\title{
OBSERVATIONS OF SPECTROSCOPIC BINARIES AT ULTRAVIOLET AND RED WAVELENGTHS
}

Francis C. Feke1, Jr.

Goddard Space Flight Center, Code 685

Greenbelt, Maryland 20771

\section{Abstract}

Recent advances in detector technology as well as the launching of the International Ultraviolet Explorer (IUE) satellite have opened new spectral regions for extensive spectroscopic observation. A program to determine the feasibility of radial velocity measurement for binaries whose secondaries are of earlier spectral type has been initiated with the IUE satellite. It is expected that velocities with uncertainties as small as $2-5 \mathrm{~km} \mathrm{~s}^{-1}$ may be obtained for some stars. This observing program includes tau Per and other composite spectrum binaries as well as several early-type binaries.

For the past five years, a Reticon solid-state photodiode array detector has been used at the coude focus of the $2.7 \mathrm{~m}$ McDonald observatory reflector to obtain radial velocities of spectroscopic as well as short-period visual binaries. The high quantum efficiency, red sensitivity, and stability of this detector system permit velocities of high accuracy to be determined and previously unseen spectroscopic components to be detected.

\section{ULTRAVIOLET OBSERVATIONS}

With the launch several years ago of the International Ultraviolet Explorer (IUE) satelitite, spectroscopic observation of this previously little explored wavelength region became routinely possible. A proposal by $C$. Harvel and myself to determine the feasibility of radial velocity measurement from observations obtained with the IUE high dispersion cameras was recently accepted by NASA. Observations during the fourth year have begun on several systems.

An examination of the literature shows that there are a number of singlelined binary systems with well-determined orbits whose secondary may be a star of earlier spectral type. This is indicated by a large mass function or the composite nature of the spectrum. In the case of a system containing a latetype giant or supergiant and a B or A main-sequence star, velocities of the early-type star are often extremely difficult if not impossible to measure because of the contamination by numerous lines of the late-type star. Lines of the earlytype star should be easily visible in the ultraviolet where the flux from the late-type star is generally lessened.

Measurement of the radial velocities of the early-type, as well as velocities of the late-type star to provide a check of these measurements, would permit the determination of the minimum masses. In cases where the orbital inclination is known from eclipses or speckle interferometry, actual masses can be determined. Low dispersion observations of the energy distributions in the far ultraviolet can give an accurate determination of the spectral type of the early-type star. Unfortunately, the IUE satellite was not constructed for the purpose of obtaining accurate absolute radial velocities. However, an examination of 
current IUE reduction and spectrum aquisition procedures suggests that velocities may be determined with uncertainties as small as 2-5 $\mathrm{km} \mathrm{s}^{-1}$ if appropriate measures are taken during observation and reduction.

One problem is that temperature drifts of the spectral format simulate a velocity shift of the entire spectrum. This shift amounts to $4.4 \mathrm{~km} \mathrm{~s}^{-1}$ per ${ }^{0} \mathrm{C}$ for the long wavelength camera. Thus, enough comparison arc calibration exposures must be obtained so that this temperature-veloctiy relation can be modeled and taken into account in the reductions.

A second difficulty is that the small aperature rather than the large one must be used so that the star and the comparison arc lamp both fill the aperature. Unfortunately, the small aperature is only about $60 \%$ as efficient as the large one so that substantial increases in exposure time may be required for faint stars. Systems on our observing list include the composite stars alpha Equ, HR 7133, and tau Per. McAlister (1978) and several other observers have resolved the latter by speckle interferometry.

Reduction of these observations has just begun. Thus, there are no results available at this time.

\section{RED AND INFRARED OBSERVATIONS}

Spectroscopic observations at McDonald Observatory of the University of Texas over the last few years with a solid-state Reticon photodiode array detector (Vogt, Tul1, and Kelton 1978) have shown that for most types of spectroscopy, the new generation of solid-state detectors will be a substantial improvement over the photographic plate and particularly useful for work on close binary and multiple stars (Feke1, Lacy, and Tomkin 1980). The Reticon can obtain observations with signal-to-noise ratios as large as several thousand to one. As a result, it can detect very broad and/or very weak spectral features of secondary and tertiary stellar components. The Reticon also has a very high quantum efficiency, $40 \%-80 \%$, over the wavelength range $4000 \AA$ to $9500 \AA$. Such efficiency is particularly important at red and near infrared wavelengths since stars of later spectral type than the primary can be more easily detected at these wavelengths than in the blue.

Since 1976, Reticon observations of a group of close multiple stars with periods $\lesssim 100$ years have been obtained with the McDonald $2.7 \mathrm{~m}$ reflector and coudé spectrograph system. The primary wavelength regions being observed are centered on $4505 \AA$ for $B$ and early-A stars and $5890 \AA, 6430 \AA, 6700 \AA$, and $8800 \AA$ for later type stars. These observations at $4.4 \AA \mathrm{mm}^{-1}$ cover a wavelength range of about $100 \AA$ and have a resolution of $0.2 \AA$ or $0.3 \AA$.

Most of these multiple systems consist of a visual binary, one or both of whose components are short-period spectroscopic binaries, making the systems triple or quadruple. In such a system it is possible to combine spectroscopic observations with visual or speckle interferometric ones to determine the parallax of the system and the masses of the components. Table 1 1ists 15 such systems being extensively observed at McDonald Observatory. As a result of the capabilities of the Reticon detector, one-third of the systems in Table 1 have had at least one new spectroscopic component detected (Feke1 1979).

Figure 1 shows a red wavelength observation of 13 Cet in which the visual secondary has been detected spectroscopically. Figure 2 shows the detection of the short-period secondary in the triple system HD 203345. Because of these new detections, the Reticon has substantially increased the number of components for which masses can be determined. 
TABLE 1

Systems Observed for Fundamental Parameters

\begin{tabular}{|c|c|c|c|c|c|c|c|c|c|}
\hline System & Spectral Type & $\mathrm{m}_{\mathrm{v}}$ & $\begin{array}{c}P_{L} \\
\text { (years) }\end{array}$ & $e_{L}$ & $T_{L}$ & $\begin{array}{c}\text { Ob } \\
\text { McD } \\
\text { (years) } \\
\end{array}$ & $\begin{array}{c}\text { servati } \\
\text { Other }\end{array}$ & ions & $N(M)$ \\
\hline $\begin{array}{l}13 \text { Cet } \\
\text { eta Ori } \\
\text { mu Ori } \\
64 \text { Ori } \\
\text { 1 Gem } \\
\text { HR } 3337 \\
\text { HR } 3551 \\
\text { p VeT } \\
\text { DL Vir } \\
\text { HR } 6497 \\
\text { HD } 165590 \\
\text { psi Sgr } \\
\text { beta Cap } \\
\text { HD 202908 } \\
\text { HD 203345 }\end{array}$ & 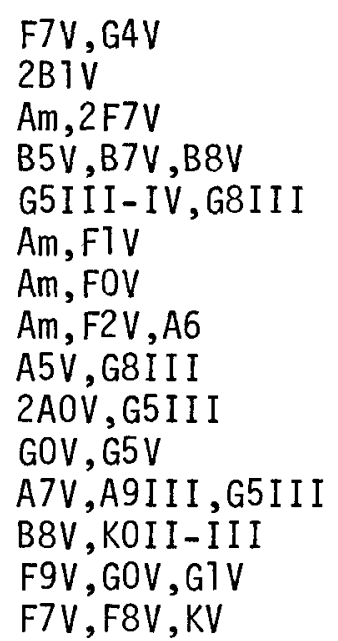 & $\begin{array}{l}5.2 \\
3.4 \\
4.1 \\
5.1 \\
4.2 \\
6.5 \\
5.3 \\
3.8 \\
7.0 \\
6.1 \\
7.1 \\
4.8 \\
3.1 \\
7.0 \\
6.8\end{array}$ & $\begin{array}{r}6.9 \\
9.2 \\
18.3 \\
13.2 \\
13.4 \\
53.0 \\
7.3 \\
16.3 \\
6.2 \\
3.3 \\
20.2 \\
20.0 \\
3.8 \\
76.1 \\
6.1\end{array}$ & $\begin{array}{l}0.77 \\
0.43 \\
0.76 \\
0.70 \\
0.32 \\
0.45 \\
0.20 \\
0.73 \\
0.46 \\
0.40 \\
0.96 \\
0.47 \\
0.43 \\
0.88 \\
0.79\end{array}$ & $\begin{array}{l}1980.8 \\
1980.4 \\
1984.5 \\
1980.6 \\
1982.0 \\
1948.4 \\
1986.0 \\
1985.6 \\
1982.5 \\
1982.4 \\
1978.6 \\
1975.1 \\
1981.8 \\
1985.5 \\
1985.8\end{array}$ & $\begin{array}{r}4 \\
4 \\
4 \\
5 \\
6 \\
7 \\
4 \\
2 \\
6 \\
5 \\
2 \\
11 \\
2 \\
5 \\
5\end{array}$ & $\begin{array}{l}\text { yes } \\
\text { yes } \\
\text { yes } \\
\text { yes } \\
\text { yes } \\
\text { no } \\
\text { no } \\
\text { yes } \\
\text { yes } \\
\text { yes } \\
\text { yes } \\
\text { no } \\
\text { yes } \\
\text { yes } \\
\text { yes }\end{array}$ & $\begin{array}{l}\text { spec, vis } \\
\text { vis } \\
\text { spec, vis } \\
\text { spec, vis } \\
\text { spec, vis } \\
\text { spec } \\
\text { spec } \\
\text { spec } \\
\text { spec, vis } \\
\text { vis } \\
\text { spec, vis } \\
\text { spec, vis } \\
\text { vis } \\
\text { spec, vis } \\
\text { spec, vis }\end{array}$ & $\begin{array}{l}1 \\
3 \\
2 \\
2 \\
1 \\
4 \\
2 \\
3 \\
3 \\
3 \\
1 \\
1 \\
3 \\
1 \\
3 \\
3\end{array}$ \\
\hline
\end{tabular}

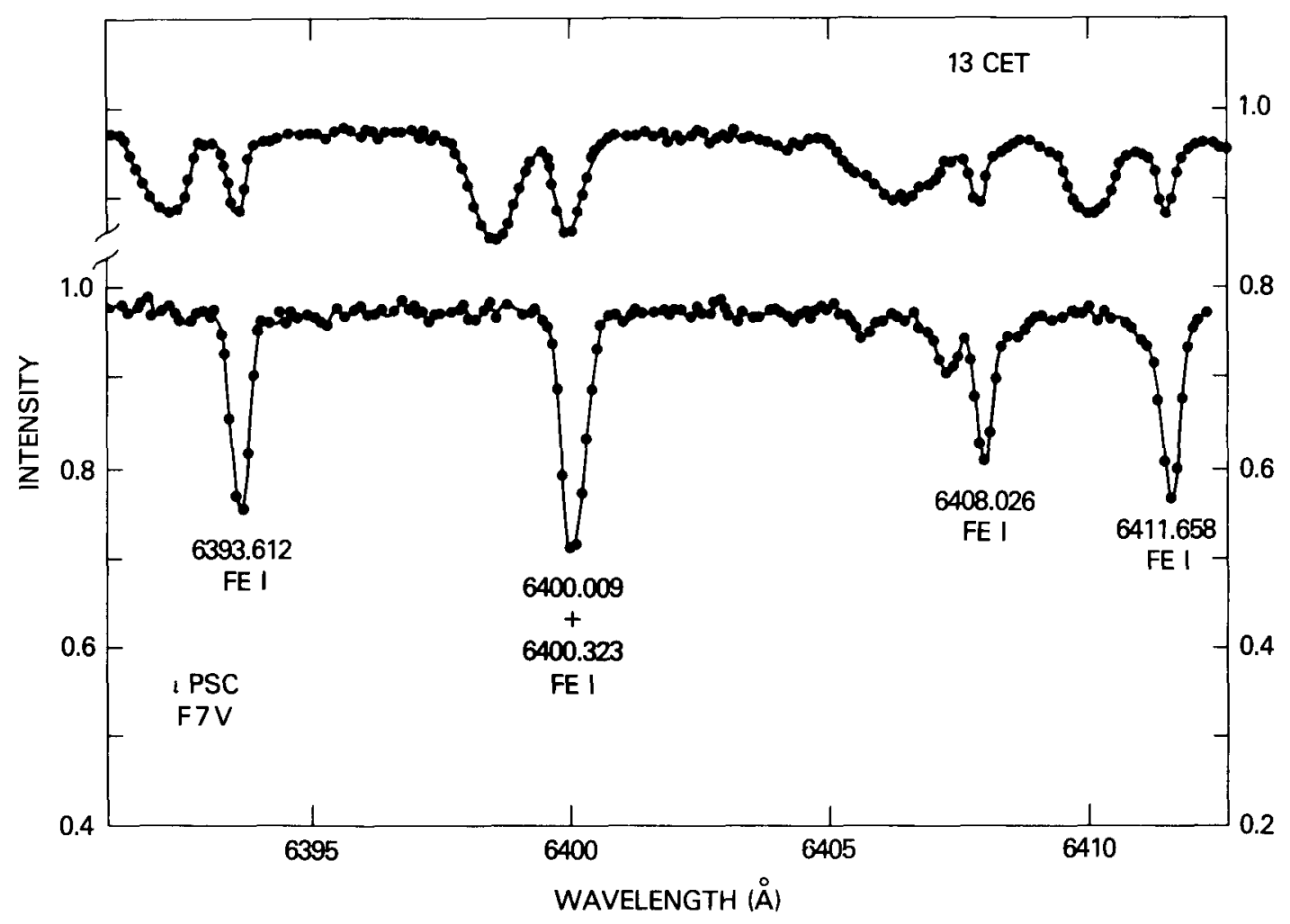

Fig. 1 A portion of a Reticon observation of 13 Cet compared with one of the late F-type star iota Psc. The broad lines of 13 Cet are of $\mathrm{Aa}$, the previously seen component, narrow lines of $\mathrm{B}$ are of the new component. 


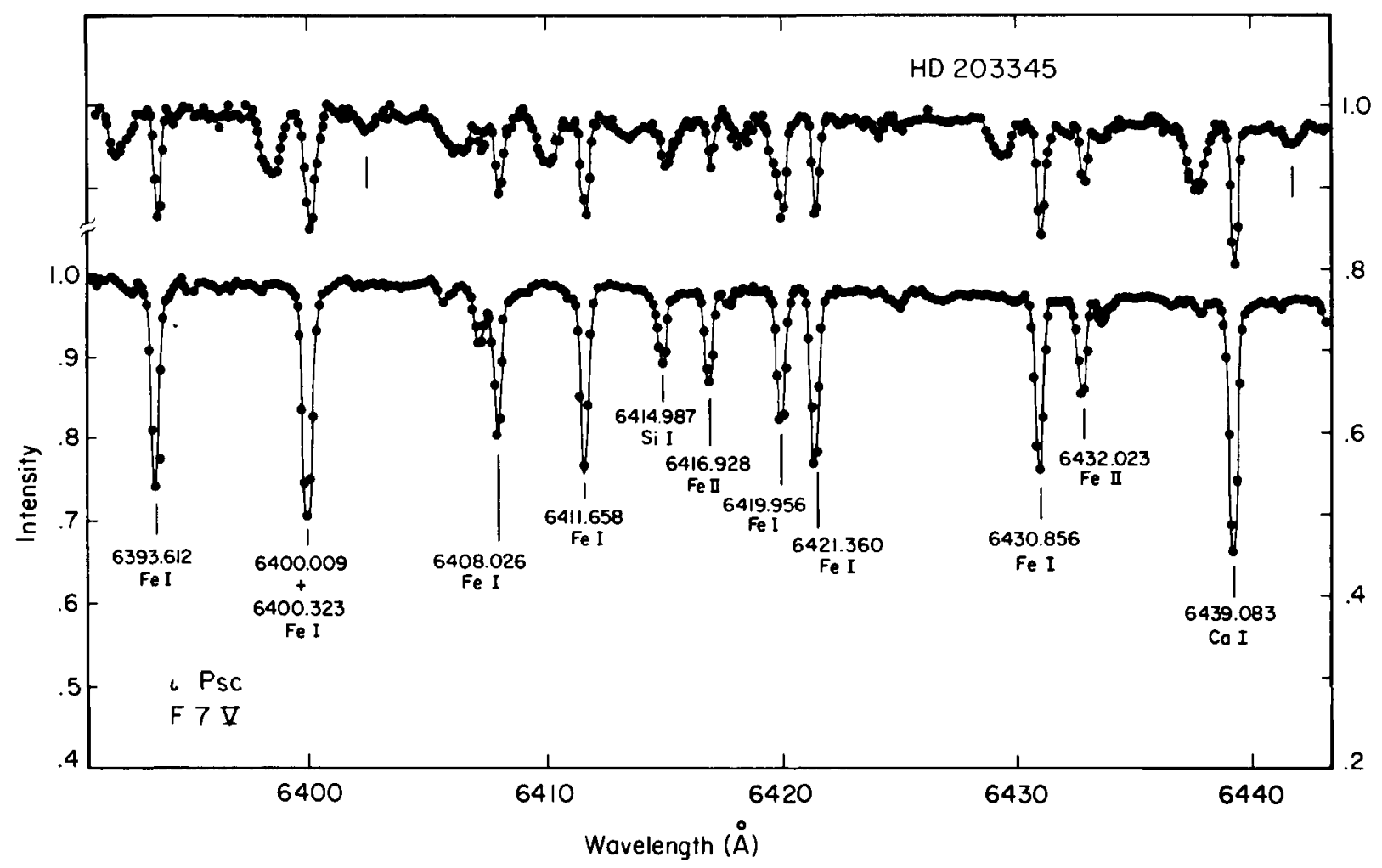

Fig. 2 A portion of a Reticon observation of HD 203345 compared with one of the late F-type star iota PSC. Lines of component B of HD 203345 are aligned with the lines of iota PSC. Lines of Aa are blue shifted and tick marks indicate the red shifted lines of $\underline{A b}$, the newly detected component.

\section{References}

Fekel, F. C. 1979, Ph. D. Dissertation, University of Texas at Austin. Fekel, F., Lacy, C. H., and Tomkin, J. 1980, IAU Symposium 88, Close Binary Stars, ed. M. Plavec, D. Popper, and R. K. Ulrich (Dordrecht: ReideT) P. 53. McAlister, H. A. 1978, Ap. J., 225, 932. Vogt, S. S., Tul1, R. G., and Kelton, P. 1978, Appl. Optics, 17, 574. 